\title{
La indagación netnográfica de dos comunidades virtuales de maternidad como espacios de significación
}

\author{
Rosario Barba González
}

\begin{abstract}
Resumen
Se parte de un recorrido conceptual sobre las comunidades de interpretación y las comunidades virtuales como espacios en los que se construyen y descifran significados. Posteriormente, se trabaja el método de la netnografía y su pertinencia para el análisis de comunidades creadas y que operan en un entorno digital. Estos dos puntos de partida se emplean para describir la discusión en torno a los grupos de maternidad en línea, para poder aterrizar en los dos casos de trabajo y las observaciones que se han realizado en ellos. Se trata de dos grupos de la plataforma Facebook con temática de maternidad. A partir de esta similitud, se observan contrastantes. Uno de los dos se encuentra anclado geográficamente y es diverso en cuanto a los puntos de vista que integra acerca de la maternidad. En el otro, la ubicación no es un factor relevante pero sí lo es la visión de la maternidad y la crianza. Para cada uno de ellos se describen algunas observaciones participantes que dan muestra de cómo estos grupos son espacios de mediación en la construcción del significado de la maternidad para sus integrantes.

Abstract

The present article sets its foundation from a conceptual journey on the interpretation communities and the virtual communities as spaces in which meanings are constructed and interpreted. Subsequently, the method of netnography and its relevance for the analysis of communities created and operating in a digital environment are studied. These two starting points are used to describe the discussion around online maternity groups, to be able to land on the two work cases and the observations that have been made on them. These are two groups on the Facebook platform with the theme of maternity. From this similarity, contrasts are observed. One of the two is geographically anchored and is diverse in terms of the views it integrates about motherhood. On the other, location is not a relevant factor, but the vision of motherhood and upbringing is. For each one of them some participant observations are described that show how these groups are spaces of mediation in the construction of the meaning of motherhood for their members.

Palabras Clave

Maternidad, Netnografía, Comunidad en línea, Red social.

Keywords

Motherhood, Netnography, Online community, Social network.
\end{abstract}

\section{Introducción}

Las comunidades son espacios de intercambio de significados y construcción de identidades. En este documento se pretende realizar la observación de dos grupos de la red social Facebook con diferentes características, aunque enfocados a la maternidad. Mediante el empleo de la técnica de la netnografía se busca observar su construcción de significados acerca de la maternidad. Se parte del supuesto de que el ingreso y la permanencia en este tipo de grupos se constituye como un espacio de reafirmación y discusión de las expectativas e inquietudes sobre el ejercicio de la maternidad. Por lo tanto, estas configuraciones comunitarias pueden ser efímeras y, no por ello, menos significativas. Las condiciones de comunicación han abierto, desde hace ya muchos años, las posibilidades para desdoblar los encuentros 
y las interacciones hacia espacios en línea, incluso procesos profundos de transformación personal y familiar como puede ser la maternidad. Este documento describe una parte de un proyecto de investigación más amplio que se pregunta por los encuentros en grupos de maternidad en la red social Facebook. Desde la bibliografía relacionada, se plantea que este tipo de agrupaciones se construyen dada la necesidad de compartir un momento paradigmático, la dificultad para encontrar grupos de este tipo en los entornos físicos y la disponibilidad del entorno virtual, entre otros. La caracterización que se hace desde los estudios de las comunidades en línea y específicamente los grupos de relación con esta temática, se desdobla en las observaciones participantes. El ejercicio se ha realizado en dos grupos diferentes, uno con un fuerte anclaje geográfico, aunque diverso en cuanto a visiones y actividades de la maternidad y otro, que abarca mujeres (y poco a poco, algunos hombres) de distintos puntos de Iberoamérica, bajo una bandera muy específica de la "crianza respetuosa". Se inicia con un recorrido conceptual sobre las comunidades de interpretación (Orozco, 1991), y las comunidades virtuales (Nor, Othman, Yusof, 2018; Ruiz, 2008), como espacios en los que se construyen e interpretan significados. Posteriormente, se trabaja el método de la netnografía (Del Fresno, 2011; Kozinets, Hemetsberger, Schau, 2008; Kozinets, 2010; Turpo, 2008) y su pertinencia para el análisis de comunidades creadas y que operan en un entorno digital. Estos dos puntos de partida se emplean para describir la discusión en torno a los grupos de maternidad en línea (Imbaquingo Pérez, 2019; McDaniel, Coyne, Holmes, 2012), y así poder aterrizar en los dos casos de trabajo y las observaciones que se han realizado en ellos. La técnica de la netnografía se utiliza para investigar la instancia especifica dónde la comunidad es producida mediante la comunicación mediada por computadora. El término usado más frecuente para referirse a este tipo de grupos es "comunidad virtual". Estos grupos sociales tienen una existencia real gracias a sus participantes y esto tiene efectos consecuentes en diversos aspectos (Del Fresno, 2011). Con estos puntos como eje, se eligieron las publicaciones para analizar y servir como ejemplo de conformación de comunidades interpretativas en virtud de compartir marcos referenciales, lenguajes e intereses en común. El trabajo se ha realizado desde la perspectiva de una observación participante en la que la autora de este trabajo se inserta por cumplir las características requeridas en ambos grupos. La inserción en los grupos se hizo antes del inicio de este estudio y ha sido la estadía en los grupos la que ha detonado el interés, tanto con la posibilidad de realizarlo. Desde la estancia en los grupos es que se permitió el acercamiento a sus integrantes. Se solicitó permiso a las administradoras de los grupos por mensaje privado. También se publicó en ambos que iba a hacer este estudio con el objetivo de comprender cómo son las comunidades en línea. En ambos se obtuvieron likes como respuesta, aunque no comentarios. Las redes sociales virtuales se han aprovechado para construir y estudiar grupos sin preocupación por el anclaje territorial. Con frecuencia ocurre en torno a un interés común de personas que no encuentran con quién compartir esa preocupación en su entorno físico cercano. La maternidad compartida es uno de los nuevos enfoques neocomunitarios. Como problema social, se plantea en relación con la configuración de formas de asociación y compromiso en los entornos de socialización virtual. Representa una dimensión bastante desconocida en los estudios de ese tipo. Saber qué está pasando con estas formas de socialización virtual en la crianza y maternidad es un gran hueco que trae aparejado espacio de saber en distintas dimensiones del saber tales como las nuevas maternidades, los estudios del capital social digital y la socialización en línea.

\section{Comunidades virtuales como espacio de intercambio simbólico}

La interpretación que se construye del mundo depende de una combinación de mediaciones que intervienen en la forma en la que se experimenta la vida. Esta combinación, a su vez, depende de los recursos disponibles a través de los cuales se realizan las mediaciones. En este afán de comprender el mundo que les rodea, se conforman comunidades de interpretación en las que los usuarios, mediante complicados juegos de mediaciones, producen sentidos y construyen identidades (Orozco, 1991). Una comunidad se refiere a un grupo de personas que comparten interacción, vínculos sociales y un formato de interacción común, local o espacio. En una comunidad se encuentran opciones de significados negociadas por los individuos en contextos de constante cambio. En ellas también se encuentra un sentido de permanencia y de contacto repetido. 
Se hallan construidas a partir de interacciones sociales y de familiaridad entre sus miembros, a partir del cual se construye la pertenencia (Nor et al., 2018; Ruiz, 2008). En este caso, interesan aquellas que se desarrollan en un espacio virtual, específicamente en redes sociales virtuales. Una de las primeras definiciones que se tienen del fenómeno, remite a agregaciones sociales que emergen en Internet cuando una suficiente cantidad de personas discute por suficiente tiempo y con suficiente emoción como para formar redes de relaciones en el espacio virtual (Rheingold, 2000, p. 5). Una comunidad en línea implica la conversación organizada y el cumplimiento de reglas compartidas. Pueden ejecutarse en los distintos entornos que provee Internet, como blogs, foros, espacios de tableros de mensajes, salas de chats o, como es el caso que interesa a esta investigación, grupos en redes sociales como Facebook (Nor et al., 2018). Una de las hipótesis más generalizadas es que las comunidades virtuales son una reverberación, a través de tecnologías digitales, de los espacios de socialización que no están necesariamente vinculados a la familia o al trabajo, llamados terceros lugares y que, si bien también existen en la realidad presencial, en Internet adquieren un potencial comunicativo y de identificación personal diferentes. Las relaciones, el intercambio y la formación de comunidades virtuales a través de tecnologías digitales, han venido a yuxtaponerse e, incluso, a sustituir las funciones tradicionales de los terceros lugares (Ruiz, 2008). Se trata de lugares de encuentro que se construyen a partir de intereses compartidos. Una de las formas de categorización más comunes responde a los temas de estos intereses: de transacción, de interés, de fantasía o de relación. Una comunidad de transacción, trata principalmente de la compra y venta de productos y servicios en línea; una comunidad de interés, tiene como objetivo un grupo de personas que interactúa con otro en torno a temas específicos como el diseño o la jardinería; una comunidad de fantasía es una en la que los miembros crean y discuten mundos inexistentes y; la de relación está formada por personas que comparten experiencias de vida en específico, como una enfermedad personal o de un familiar. Estas últimas pueden generar vínculos profundos (Nor et al., 2018). Las comunidades virtuales de este trabajo, poseen lenguajes, significados, causas y culturas reales. "Estos grupos sociales tienen una existencia real para sus participantes $\mathrm{y}$, por lo tanto, tienen efectos en muchos aspectos de su comportamiento" (Kozinets, 1998, p. 366). Las comunidades que se analizan en esta investigación son de este último tipo, a partir del hito de vida que supone la maternidad. Las comunidades virtuales están compuestas, principalmente, de personas que raramente se conocen cara a cara, quienes son medianamente responsables de la información que comparten y cuya identidad quizá quede permanentemente anónima. Esto porque el ser virtual está separado de cuerpo físico y así, aparentemente, de consecuencias materiales. La identidad en Internet es construida, múltiple, descentrada y un trabajo en constante progreso (Ruiz, 2008). Una forma de explicar el fenómeno de la configuración de comunidades virtuales es a través de la noción de cultura participativa. Esta sugiere un cambio en el papel de los usuarios de Internet, así como del entorno virtual y de las comunidades virtuales que ahí se forman y desarrollan. Una actuación más activa es requerida, tanto para producir como para consumir. Internet tiende a volverse un medio en el que esta actividad es posible y habilitada (Jenkins, 2006). Mientras se crean y comparten contenidos, se pueden crear lazos sociales y proponer una diversidad de expresiones culturales (Jenkins, 2009). Así, en las comunidades virtuales como las que aquí se analizan, se presentan las cuatro categorías que Jenkins (2006) sostiene sobre cultura participativa: afiliación al grupo; formas de expresión a partir de la reproducción del lenguaje compartido y que emplea herramientas del entorno de Internet; generación de espacios que fomentan la solución colaborativa de problemas y la contribución a la circulación de la información de interés para esa comunidad. A raíz de estas cuatro características, en la vivencia cotidiana, las comunidades virtuales son pensadas como lugares de pertenencia, en donde es posible construir arraigo y autorreconocimiento. Por lo tanto, la convivencia entre los integrantes se experimenta, efectivamente, como una forma de integración a un grupo con el que se comparten visiones del mundo, hábitos, creencias y valores (Imbaquingo Pérez, 2019). Para el estudio de las comunidades se parte de que su existencia es una forma de apropiación de Internet, en particular, de la red social virtual Facebook, la plataforma de red social líder de la actualidad. En abril del 2020 superó los 2,600 millones de usuarios activos diarios (Moreno, 2020). Fue la primera plataforma en superar los mil millones de usuarios. México es el quinto país que 
más usuarios aporta a la plataforma, con 54.6 millones en 2018. La distribución de los usuarios muestra una paridad de género casi exacta y una concentración en edades entre los 18 y los 44 años, sobre todo en el rango entre los 25 y $\operatorname{los} 34$ años (Statista, 2020). En un país en el que 86El perfil de la plataforma se enfoca en el intercambio entre familia y amigos por lo que empuja las interacciones por características como fotografías, estatus y juegos sociales. Redes sociales como Facebook se consideran integradas con las formas en las que los usuarios valoran y practican sus relaciones sociales y comunitarias, ya que perciben a la plataforma "simplemente" como un elemento más de sus vidas (Dalsgaard, 2016). Estas características ocasionan que los miembros de estas comunidades sean ubicuos y el conjunto es irrepresentable. Quiere decir que la comunidad virtual se identifica simbólicamente con cualquier o ningún lugar del mundo o dominio lingüístico. Ello es posible por los sistemas de comunicación digital que imponen un ritmo diferente a los intercambios en las comunidades virtuales y que, con ello, provocan una mutación en las formas de relacionarse, tales como las conversaciones paralelas en pantallas simultáneas, los diálogos caóticos, o los intercambios masivos vinculados al hipermedia (Ruiz, 2008). En este punto, no sobra la insistente aclaración de que todo el mundo tiene acceso a la red: la mayoría de la población mundial permanece ajena, preocupada por la supervivencia diaria. Sin embargo, aquí y con base en las reflexiones tejidas hasta ahora, se considera a la significación como un proceso social en la medida en que cada miembro participa de una cultura determinada y está sujeto a una serie de relaciones, entonces, es en la comunidad donde se produce el sentido (Orozco, 2012). Así, aunque las comunidades presenciales siguen siendo predominantes en el mundo, y con las que se identifica la mayoría de las poblaciones, las comunidades virtuales, así como el espacio de los flujos en las que se instauran y del que provienen, expresan la lógica social en la sociedad de la información (Ruiz, 2008). Debido a la constante presencia de las redes sociales virtuales en la vida de los usuarios, estas plataformas tienen, decididamente, un fuerte impacto social. Las barreras son cada vez más borrosas entre la vida offline y la vida virtual. Por lo tanto, con el objetivo de caracterizar las prácticas que conforman las comunidades virtuales en Facebook, sus interrelaciones comunicativas, contradicciones e interacciones en la vida presencial de las mujeres en etapa de maternidad y crianza, se ha hecho uso de las técnicas comprendidas en el método de la netnografía. En el siguiente apartado se detalla su pertinencia para el análisis de este tipo.

\section{Netnografía y análisis de comunidades}

Para el análisis de grupos de crianza y maternidad que usan tecnología para compartir y discutir puntos de vista e información, destaca la técnica de etnografía virtual (Hine, 2004) o netnografía (Bowler, 2010; Del Fresno, 2011; Kozinets, 2010; Turpo, 2008). Estas técnicas han sido desarrolladas a partir de la necesidad de comprender la extensión de la vida cotidiana hacia los entornos en línea. Se parte de pensar cinco características que distinguen las comunidades virtuales de aquellas con base territorial. Se habla de que están desterritorializadas para describir que los integrantes no necesariamente se encuentran en el mismo espacio entre ellos ni el etnógrafo que realiza la observación. En ese sentido, el espacio geográfico es contingente pero no determinante. Es decir, el cuerpo y territorio existen, pero estos dos factores no determinan la existencia de la comunidad, sino que es guiada por relaciones. En la mayoría de los casos, la comunidad se identifica con un lugar específico del mundo o con un dominio lingüístico. El cuarto punto describe la forma en la que los intercambios comunicativos se modifican dadas las condiciones de los sistemas y plataformas. Por lo tanto, se pueden encontrar conversaciones paralelas, diálogos no recíprocos y desordenados o intercambios masivos. Finalmente, la interacción tiene pocos lugares referenciales fijos y poca inercia (Ruiz, 2008). Frente a esta avalancha que aparece como desordenada, Internet puede proporcionar metodologías y técnicas para la obtención de datos, el análisis y la interpretación, así como la propia lectura de textos etnográficos (Ruiz, 2008, p. 6). Una de las técnicas que destacan es la observación, sobre todo la participante. La capacidad de análisis de la técnica se pretende potenciar aquí con el análisis del proceso discursivo o el "textualismo", es decir, los mensajes de texto intercambiados por las integrantes de la comunidad (Imbaquingo Pérez, 2019, p. 12). Tradicionalmente se ha empleado a Facebook para el análisis de redes y la caracterización de perfiles de usuarios. Este estudio se pregunta por el potencial que tiene la plataforma para rastrear construcciones comunitarias entre usuarios que 
comparten intereses particulares. En este sentido, apunta hacia la colectivización o la búsqueda de ella en el espacio virtual. Busca comprender cómo es que Internet se ha constituido en un espacio para tejer redes y conexiones que aprovechan la tecnología. Se implementó una metodología netnográfica en dos grupos cerrados de Facebook: Crianza Fisiológica 2 y Las nuevas mamás Qro. Ambos grupos sirven como espacios para compartir información sobre crianza y maternidad entre sus miembros. Sin embargo, difieren en su aproximación a esta etapa, anclaje territorial, intereses, puesta en marcha y reglamentación. La netnografía puede ser definida como el reporte escrito de los resultados de un trabajo de campo estudiando las culturas y comunidades que surgen online, mediante una computadora o en comunicaciones basadas en internet, dónde el reporte escrito y el trabajo de campo están metodológicamente informados por las técnicas tradicionales de antropología cultural (Kozinets, 2010). Se propone aprovechar la modalidad de los grupos de la plataforma Facebook, que permite abordar esas otras maneras de reconstituir las relaciones en línea. Considerar sus contenidos como fuentes de información supone tensionar la idea de presencialidad física del investigador en el campo y asumir el desafío de afrontar nuevas experiencias metodológicas y problematizaciones derivadas de su utilización y de su legitimidad. En Facebook, los participantes no sólo proveen comentarios de sus prácticas, sino que las discuten, las ejemplifican, se burlan o se suscriben a lo que otros expresan. Todo ello implica una negociación de significados que se lleva a cabo mediante la producción, intervención o la distribución de contenidos visuales, audiovisuales y textuales. Los comentarios permanecen registrados en la plataforma $\mathrm{y}$ en los grupos, de manera que es posible tener un acceso compuesto a sus discursos, entre cruzarlos y contrastarlos incluso a través del tiempo. Todas estas posibilidades se encuentran fácilmente accesibles a los participantes gracias a las características de la plataforma. Más interesante, los usuarios las tienen aprendidas y normalizadas así que las emplean de manera orgánica, lo cual puede ser sumamente provechoso para el proceso de investigación (Capogrossi, Magallanes, Socaire, 2015; Dalsgaard, 2016). Facebook ofrece la posibilidad de examinarlos recurrentemente, así como tener un registro automático de las interacciones a lo largo del tiempo. De tal suerte que los registros de los grupos y páginas de Facebook permanecen disponibles para su revisión en cualquier momento. Es así como se convierte en un entorno privilegiado de análisis sobre la construcción $\mathrm{y}$ el desenvolvimiento de las comunidades que se generan e integran en línea. Por ejemplo, las que se construyen en torno a momentos radicales de la vida humana como las de maternidad.

\section{La maternidad en redes}

La maternidad (y la paternidad, aunque este proceso específico exceda la reflexión que aquí se plantea) se presenta como un largo proceso de reestructuración que comienza con el embarazo y continúa por un tiempo después del parto. En otros momentos de la historia humana, las exigencias de la maternidad no suponían el nivel de contradicción para la mujer que implican ahora, ya que su función se desarrollaba como centro de la vida doméstica. Actualmente, con más mujeres presentes en la esfera pública y de producción, se enfatizan las demandas de la mujer hacia la vida privada, sobre todo en los primeros meses de vida del nuevo integrante del núcleo familiar. Se trata de una cuestión irreconciliable que casi siempre termina por la renuncia de la madre a la vida pública, aunque sea por el mínimo de 40 días que requieren, en México, las guarderías. Lo anterior, con las posibles consecuencias que ello puede implicarle. Se trata de un desafío no sólo desde la estructura productiva sino también desde la simbólica pues se cuestiona su papel como "mujer independiente y poderosa" frente al de la "buena madre". Ambas son construcciones significativas colectivas con expectativas de gran peso en las decisiones individuales. Uno de los resultados es que, a consecuencia del parto, la mujer vive una exclusión de los sistemas sociales de los que formaba parte. Esto incluye su espacio laboral, pero también las amistades que se reúnen en lugares de esparcimiento a los que rara vez se integra un recién nacido. Con la familia se pueden llegar a estructurar una serie de conflictos a partir de las brechas generacionales y las posturas y acciones que se requieren para ejercer la maternidad. De esta manera ocurre un descentramiento que genera en las mujeres un sentimiento de soledad y que se refuerza con la disonancia entre esfera pública y la nueva privada en la que se encuentra la mujer llena de interpelaciones. Dadas las exigencias que la nueva vida impone sobre la familia y sobre la madre, la maternidad es uno 
de los eventos más estresantes en la vida (Imbaquingo Pérez, 2019; McDaniel et al., 2012; Orton-Johnson, 2017, entre otros). Frente a estas tensiones, las características digitales del medio y el intercambio de contenidos permiten que las madres expresen sus necesidades y deseos propios, que en ocasiones pueden ser preocupaciones por el bebé, pero en otras pueden ser independientes. Es un espacio en el que se permite el ir y venir entre el rol que están construyendo y el anterior, que frecuentemente se percibe como el real frente al que están inventando para enfrentar la maternidad (Imbaquingo Pérez, 2019). Ha sido ampliamente establecido que el apoyo social absorbe el estrés de la maternidad. Los grupos de Facebook son un medio bastante popular para que las madres se conecten con una comunidad más amplia que su entorno directo. Esta integración contribuye a la percepción de acompañamiento social y la reducción de la tensión lo que, a su vez, contribuye a mejorar otros aspectos del bienestar de la mujer, que incluyen percepciones positivas de la calidad del lazo matrimonial y la reducción del conflicto en esta relación, así como la disminución de síntomas depresivos (McDaniel et al., 2012). Así, se han encontrado distintas consecuencias al tiempo que las nuevas madres pasan frente a las pantallas de Internet. Para algunos, se trata de espacios de empoderamiento a partir de la divulgación de información y la interacción de experiencias compartidas. Otros, sin embargo, ven en la red lugares de reafirmación de las normas tradicionales sobre la feminidad y la familia. En estos espacios se reproducen las exigencias que recargan el trabajo del hogar en la madre (McDaniel et al., 2012). Los primeros describen la experiencia de mujeres que aprovechan los distintos foros de internet para buscar acompañamiento frente al sentimiento de soledad producido por las afectaciones que la maternidad trae a la estabilidad en su identidad. Estas mujeres refieren la necesidad de vincularse con sus iguales, que ya no son las relaciones que tenían hasta el momento, sino que van asombrándose al descubrirse pares de otras madres. Esta necesidad recae en la sensación de que sólo quien se encuentra atravesando por un proceso similar puede comprender su estado anímico. Un grupo de interacción digital cumple funciones como espacio estratégico en el que se integran, discuten y visibilizan diversos discursos más o menos cercanos al asunto de la maternidad, que, al ser tan diversa, puede llegar a incluir una enorme cantidad de temas (Imbaquingo Pérez, 2019). Al final, la maternidad se entiende también como una construcción discursiva cargada de significados que norman y regulan el comportamiento materno y la forma en que se experimenta. En los grupos de maternidad en Facebook, como en otros espacios, parte de lo que está en juego es el deber ser de una madre y, más aún, una "buena madre". Estas concepciones también ejercen una carga en la forma en la que la madre enfrenta el proceso de serlo. La idea que se plantea aquí de comunidad como una serie de redes de lazos que proveen apoyo, información, identificación, identidad, información, etcétera, resulta útil para comprender la forma en la que Internet y una buena parte de la diversidad de sus espacios se han introducido en la significación de la vida cotidiana. Una comunidad digital de maternidad responde a la necesidad de legitimar los cambios y sentimientos de la maternidad. A través de la comunicación se desarrolla un proceso de mediación (Martín-Barbero, 1987) que se genera conforme se da el intercambio de mensajes y comentarios en el grupo. Es así como la comunidad se constituye como un espacio de significación y apropiación de discursos sobre la maternidad e inciden en la forma de experimentar la crisis de identidad que viene aparejada del momento. En estos entornos se discuten, ejemplifican, negocian, burlan, reclaman y pelean las prácticas concretas que la madre instrumenta para ser una buena madre desde su propia definición de "buena madre". Existen discusiones casi dicotómicas e irreconciliables que son recurrentes en casi todos los grupos de maternidad. Estos temas incluyen: lactancia/fórmula, edad y método de introducción a los alimentos sólidos, forma y tipo de escolarización, permanencia de los hijos frente a las pantallas (a menor tiempo, mejor madre), tipo de crianza respetuosa/tradicional. A estos se agregan otros dependiendo de la especificidad del grupo, por ejemplo, el uso de medicina alternativa. Las comunidades virtuales son nuevos espacios de experiencia comparativa llevada gracias a los medios digitales. La red sociodigital, permite cierto nivel de participación las "reacciones" de gusto y disgusto pueden tener múltiples interpretaciones a las que el investigador, en principio, tiene acceso por medio de la participación con el entorno. En el análisis de su uso se reflexionan las circunstancias específicas y las condiciones en las que usan sus intercambios comunicativos. Es decir, que se examinan los contextos, las compañías, la atención, los comentarios y las opiniones que les generan. Además, permiten tener una amplia variedad de 
visiones, dada la cantidad de personas que se vinculan por medio de estos grupos (Kozinets, 1998). El alcance de los grupos en la vida individual y social se ve limitado por los procesos que se aparejan a la maternidad. Al tiempo que la madre percibe que recupera las posibilidades de reintegrarse a los círculos sociales a los que pertenecía o de construir nuevos, la interacción con la comunidad disminuye, desaparece o se transforma (Imbaquingo Pérez, 2019). En este punto, vale la pena recordar y dar voz de las dudas que se tienen frente al análisis de los grupos de Facebook pensados como comunidades. La inconsistencia de las interacciones y la falta de reciprocidad directa puede ser motivo de cuestionamiento sobre el valor comunitario de estos espacios. Sin embargo, a partir de la cultura participativa (Jenkins, 2009) se reconoce que es por medio de la colaboración mutua que los miembros de un grupo perciben que son partícipes y que son relevantes para la comunidad. No necesariamente se da una reciprocidad directa, sino que se asiste a un miembro con la confianza de que la comunidad estará para responder ante cualquier solicitud de ayuda. Es a partir de esta solidaridad que se van construyendo lazos de colaboración y de pertenencia. Estos lazos se arman y exhiben de distintas formas. Por ejemplo, en una de las comunidades que se investigan, se llegó a diseñar y portar una pulsera con la cual identificar a los miembros fuera del espacio virtual. Esta pulsera ofrecía descuentos de las empresas de las mujeres que son miembros hacia las otras que se identificaran como tales, a manera de que la identificación y la solidaridad trascendiera la pantalla hacia el entorno físico. Este es sólo un ejemplo de la numerosa cantidad de muestras de vitalidad comunitaria que se han observado en el ejercicio netnográfico. Se trata de un ejercicio exploratorio de observación participante desde el punto de vista de una madre primeriza que ingresó a los grupos a los pocos meses de ser madre y, coincidentemente, después de algunos meses de la creación de ambos. La curiosidad por el fenómeno surge, precisamente, en la vivencia, aunque la observación sistemática se realiza a partir de la solicitud de permiso en ambos grupos. La observación se realiza, entonces, sin una hipótesis como punto de partida, con la intensión de observar entornos comunitarios y en línea y las interacciones entre sus participantes, que no han sido observados antes. Se seleccionaron dos grupos de crianza y maternidad que difieren en puntos de vista y actitudes. Son similares en tanto que se trata de dos grupos comprometidos con buscar y compartir información respecto a la crianza, sin embargo, describen y se constituyen como mediaciones diferentes en la construcción de la noción de maternidad. Uno tiene un fuerte anclaje geográfico tal que excluye aquellas aplicaciones a ser miembro que no cuenten con la condición de radicar en la ciudad. Al otro no le interesa el origen de la solicitud, por lo que sus miembros son de alrededor del mundo con la coincidencia de hablar en español. Las visiones de una "buena madre" en el primero son bastante amplias. No es necesario coincidir en este sentido. En el segundo, sin embargo, se impulsa una visión de la crianza respetuosa, con una serie de implicaciones que se desarrollarán más adelante. Cada uno de ellos se construye a partir de sus ejes de interés. Los miembros defienden las posturas y los administradores ejercen la censura necesaria para que no haya demasiadas desviaciones con respecto de sus objetivos. A continuación, se describen las observaciones en cada uno de ellos. Se reservará el nombre de los dos para proteger la privacidad de sus miembros, sobre todo considerando el nivel de intimidad que se mantiene para tratar temas relacionados con la maternidad y la crianza. Se complementan las descripciones con las capturas de pantalla de algunos casos representativos. En todos ellos, se han eliminado cualquier fuente de identificación que ponga en riesgo la privacidad de los involucrados.

\section{Grupo con anclaje geográfico y diversidad de concepciones de maternidad}

Se trata de un grupo secreto creado para ser "un espacio de apoyo para nuestra labor del día a día. Poder platicar, aconsejar, recomendar y hasta ofrecer servicios o productos y así aprovechar que estamos en la misma ciudad y activar un poco la economía familiar", de acuerdo con la descripción del grupo. Las 7,285 participantes del grupo deben cumplir con tres características: ser madres o estar embarazadas, vivir en la ciudad de creación del grupo que forma parte del nombre y no estar en más de 15 grupos. Con ello se pretende que las discusiones no excedan el interés del grupo en temática ni ubicación geográfica. La última característica es un intento por controlar el ingreso de perfiles falsos, fraudulentos o con intensión exclusiva de venta. El grupo es definitivo en el primer requisito: no se admiten hombres. 
En un grupo como este, el espacio geográfico real se convierte en un centro cualitativo capaz de generar segregaciones y exclusividad. Esto se encuentra anclado al lenguaje y en personajes, historias y lugares que se comparten en la comunidad (Ruiz, 2008). Las miembros del grupo se han preocupado por indagar con quién conviven en la comunidad. En la Ilustración 1, se presenta la encuesta y las respuestas sobre la dedicación de las madres del grupo. Si bien no es una muestra representativa de las más de siete mil mujeres que están en el grupo, incluso la formulación y las opciones dan idea de la formación del grupo. En los comentarios de confirmaban las respuestas con emoticones y en muchos casos se exponía la respuesta agregando referencias de orgullo.

\section{Ilustración 1. Caracterización de las miembros del grupo a partir de una encuesta realizada al interior \\ 16 de marzo \\ Me ayudan a hacer una encuesta? \\ Quiero saber en este grupo de mamás una estadística para poder \\ entender muchas de las respuestas que damos. \\ Puedes poner tu reacción si eres \\ 4 mamá ama de casa \\ -mamá trabajo institución, empresa,etc \\ - Emamá con negocio propio \\ ¿mama trabajó en casa ventas, catálogo etc \\ O.0 534

Fuente: publicación en grupo de Facebook, 16/03/2020.

El reglamento se basa en el respeto y la autorregulación.

Se admiten ventas los lunes y viernes de cada semana con la condición de que los productos muestren sus precios en la publicación y sin que haya "competencia desleal". En caso de que no se cumplan, las administradoras y moderadoras reservan la posibilidad de ser eliminada del grupo. Las miembros del grupo también participan del cumplimiento de este requisito. En el caso que se muestra en la Ilustración 2, la publicación fue editada para incluir la descripción.

Ilustración 2. Ejemplo de la participación de los miembros en el cumplimiento del reglamento

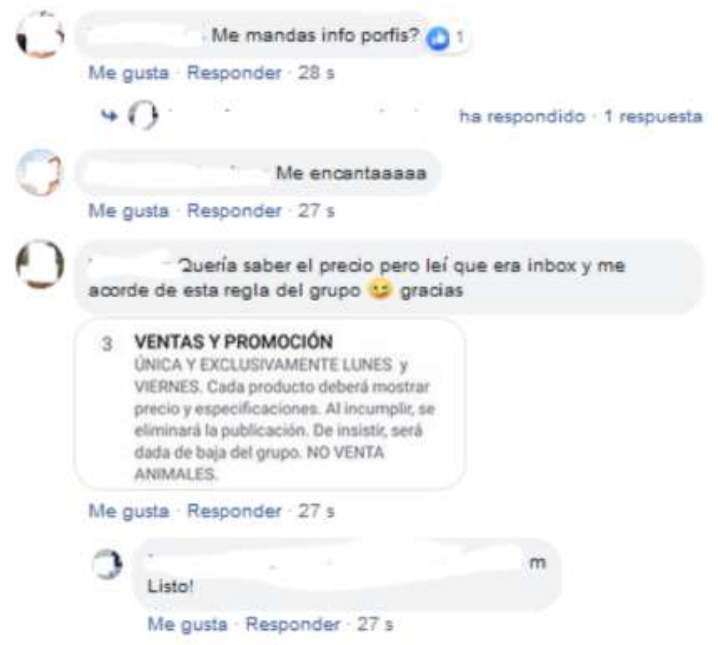

Fuente: comentarios de la publicación de Facebook,

$11 / 11 / 2019$

En este grupo no hay alguna orientación desde la cual se aporte a las dudas de las participantes. Cualquiera puede responder desde su experiencia o información recibida de cualquier fuente. Muchas de ellas referencian fuentes médicas, pero sobre todo se remiten a su experiencia. Los cursos de crianza que se promocionan o recomiendan tienen múltiples fundamentos: coaches, orientadores, distintas disciplinas de la medicina, religiones, modelos pedagógicos, consejos familiares, entre muchos otros. Frente a esta situación, el reglamento ha incluido un lineamiento sobre responsabilidad social en el que se lee que únicamente se admiten publicaciones desde fuentes oficiales para el caso de noticias, comunicados y avisos. Dada la contingencia sanitaria de la primera mitad de 2020, las administradoras han sido vigilantes del cumplimiento de esta norma. Muchas de las publicaciones piden recomendaciones para mandar a hacer una diversidad de productos o servicios, incluidos los médicos. Otras publicaciones tienen fines filantrópicos, sobre todo para ayudar a madres en situaciones precarias o de violencia. Algunos más son exposiciones de percepciones o experiencias, algunas de cotidianeidad doméstica y otras que se presentan como asuntos graves que deben ser dados a conocer en el grupo. Por ejemplo, ha habido avisos de asaltos o tentativas de asaltos para prevenir a las demás a no transitar por algún camino o carretera. En todos estos casos, las publicaciones son respondidas con mensajes de alarma tanto como de apoyo por la experiencia. Frecuentemente se leen publicaciones en las que 
se agradece al grupo y a sus miembros su apoyo e integración. Estos provienen tanto de las administradoras como de las integrantes. También se agradecen las recomendaciones de productos y servicios y se emplea el agradecimiento como estrategia de venta. Entre las dudas compartidas son medianamente frecuentes las referentes a las relaciones vecinales. Por el tema, sobre todo se trata este tema cuando se presencian casos de maltrato infantil. Claro está que las más comunes corresponden a las concernientes a las relaciones con los hijos y esposos, problemas con las familias nucleares y extensas y de cansancio por las exigencias de la maternidad. Destaca para ser trabajo de otro análisis, el imaginario compartido sobre el papel del esposo en la dinámica familiar. En comentarios, publicaciones textuales y memes ha sido referido abundantemente como un hijo más, como subordinado a las instrucciones de la madre, infiel o sujeto de infidelidad. Las dudas de salud son especialmente habituales. En la mayoría de los casos, se describe la molestia propia o de los hijos y se anexa una fotografía. Las respuestas son, casi siempre, las mismas: recomendaciones de medicamentos no controlados, recomendaciones de pomadas y terapias alternativas de marcas vendidas por distribuidoras e indicaciones para ir al médico. Con frecuencia, estas se presentan combinadas. Las publicaciones en general incluyen uno de los elementos discursivos que anuncian la pertenecía a un grupo y a la reconstrucción de la persona que se mencionaba previamente. En un grupo de este tipo es sumamente habitual la colectivización a través de un término como "mamis" "mamás" o el anglicismo "moms" y cualquier término similar. Esto antepone la característica vinculante a cualquiera otra que puedan tener las integrantes del grupo. Ese grupo representa un ejemplo del polo de lo común. Ahí no hay exclusiones ni en temática ni en aproximación a los temas de interés. El abordaje que se realiza a los mismos se construye desde discursos de lo "normal" y lo "común", es decir, desde posturas hegemónicas. Temas que en otros grupos de afinidades más específicas resultarían polémicos, aquí no se discuten. Por ejemplo, las vacunas. En un grupo así, la cartilla de vacunación está dada y no se somete a ningún tipo de reflexión o discusión. Las instrucciones oficiales de distintos organismos gubernamentales y privados se siguen y persiguen como la medida del bien actuar. El discurso de instituciones civiles y religiosas está normalizado y se asume como compartido y como parámetro del bien actuar. La mediación del deber ser de la madre se asume desde estas posturas hegemónicas. Los debates binomiales que ocurren en casi todos los grupos de maternidad también acontecen aquí, aunque de manera muy esporádica y, tanto los miembros como las administradoras, llaman al respeto de la diversidad del ser madre. Esto no es tan evidente en el caso del otro grupo construido en torno a la crianza respetuosa y fisiológica. En él, incluso estos términos articuladores son discutidos constantemente y, como se describe a continuación, el debate es más intenso

\section{Grupo con anclaje temático y diversidad de orígenes geográficos}

Una categoría de "buena madre" que se ha encontrado en grupos como los que ahora se describirán ha sido descrito como maternaje intensivo (Imbaquingo Pérez, 2019). En esta postura se percibe a la madre como un sujeto que cumple, además de un deseo personal, un mandato colectivo de continuidad de la especie, por lo que su cuerpo de mujer embarazada adquiere una cualidad significativa de compromiso con esta función social. Este grupo ha sido creado en torno a la filosofía de una especialista en musicoterapia y eutonía. Su base es la aplicación de los principios de esta última en recién nacidos y su combinación con la filosofía pedagógica Pikler. Se trata de una especialista reconocida y ocasionalmente es invitada a diálogos en radio y televisión argentina como coach de crianza. El grupo de Facebook se ha construido con seguidoras de las propuestas de la fundadora. En él se comparten dudas de crianza, que pueden ser puestas a consideración por vía, precisamente de Melina a modo de anonimato. Las respuestas que se ofrecen tienen que ver con esta visión que, en el grupo, se refiere como crianza fisiológica, así como otros compendios de las pedagogías Montessori, Waldof y Pikler. Esto es fundamental para el desarrollo de las intervenciones pues todos los participantes vigilan que no se recomienden acciones fuera de los fundamentos compartidos, así como que las preguntas se realicen en torno a la temática de interés. La creadora revisa las denuncias hechas por los participantes y resuelve conflictos. Frecuentemente responde a las dudas desde su posición de autoridad en el tema. Se trata de un grupo cerrado con 32,375 participantes, al que pueden integrarse hombres, pero la generalidad son mujeres. La 
mayoría son madres, aunque también participan educadoras, abuelas y otros roles. Es un grupo de alcance internacional por lo que sus participantes provienen, principalmente, aunque no de manera exclusiva, de Iberoamérica. Las reglas de convivencia puestas por la creadora son rígidas. $\mathrm{Su}$ principal propósito es que el grupo no se desvíe del tema de la crianza fisiológica. No se admiten ventas ni propaganda excepto en publicaciones específicas que la fundadora realiza y únicamente pueden ser propuestas de productos y servicios en torno a la temática del grupo. Los castigos son los que permite la plataforma: censura del comentario o publicación y expulsión del grupo. La crianza fisiológica combina los principios de posturas como la crianza con apego o la crianza natural, el movimiento libre y las filosofías pedagógicas Pikler y Montessori con un compromiso que, desde otros grupos de temas similares se califica como demasiado estricto. La fundadora ha recibido lo que ella ha descrito como agresiones por las exigencias que su postura supone para la maternidad y paternidad. Se la ha descrito como fundamentalista. Los participantes se encuentran en discusiones con frecuencia debido a estas exigencias. Este grupo se asume como un grupo de crianza respetuosa, lo que ha sido explorado por Villanueva (2017) como una postura basada en un discurso ecologista que se vincula con el saber experto basado en datos científicos para incidir en las decisiones de las mujeres madres. Usando como referencia este discurso, muchas madres han resignificado el vínculo entre madre e hijo en búsqueda de una experiencia "natural". De esta manera corresponde con las comunidades de enfoque ecosocial de mujeres que trascienden la maternidad para concentrarse en el papel político ecológico (Navarro Casillas, 2016). En este afán, muchas mujeres del grupo hacen publicaciones y comentan replanteándose las posturas hegemónicas con respecto de, por ejemplo, las técnicas hospitalarias y la medicalización del parto que se califican como innecesarias. En esta postura naturalista, las mujeres madres ven en estos procesos una pérdida de poder sobre su cuerpo por lo que buscan dar luz de manera natural, muchas veces en casa, sin médicos y con partera. La base científica se construye a partir de un tejido ecléctico de saberes de la psicología, la nutrición y la pediatría han colocado a la madre como figura central en el cuidado de los hijos e hijas. El grupo que se describe en este apartado se postula a sí mismo, dentro de estas nociones, como defensoras de la experiencia consciente de la maternidad, en contra de la medicalización excesiva que entorpece el control sobre el cuerpo (Imbaquingo Pérez, 2019). En la descripción del grupo se asienta esta visión que media el comportamiento y las interacciones de los miembros (Ilustración 3). Este enlista las acciones que reclama como como formas saludables y naturales de entregar amor y afecto a los hijos que incluyen, entre otros, el colecho, la lactancia prolongada, el porteo ergonómico, y el control de esfínteres guiado por el niño.

\section{Ilustración 3. Descripción del grupo con anclaje temático}

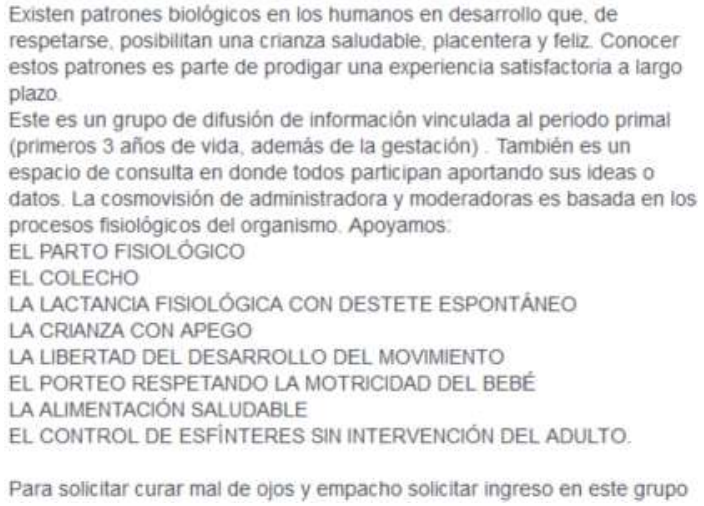

Fuente: descripción del grupo, 17/06/2017

Estos grupos se convierten en un espacio de cuestionamiento y resignificación de la maternidad y la "buena" maternidad. En él, se condensan y solidifican objetivos políticos que, asumiendo la premisa de "lo personal es político" y "el cambio comienza en casa" se proponen un cuestionamiento de las condiciones del mundo en general. Asumen que si la crianza es "mejor", la humanidad podrá dirigirse a un mejor estado (Imbaquingo Pérez, 2019). Cuestionan, por ejemplo, la organización "adultocentrista" de la sociedad en la que el niño es considerado una persona que será, no que ya es. Por lo tanto, a su parecer, no se le concede el mismo valor o consideración. Observan y, generalmente, repudian esta predominancia de los intereses de los adultos por encima de los del niño en los entrenamientos conductistas para dormir, comer, dejar el pañal, leer, moverse, dibujar, etcétera. Rechazan de manera radical todo lo que implique "dejar llorar" al niño. Por lo tanto, muchas de las publicaciones tienen que ver con las quejas de estas observaciones, sobre todo en sus entornos directos. 
Ilustración 4. Ejemplo de queja sobre el 'adultocentrismo' y la respuesta de la fundadora del grupo

9 de agosto de 2019

Hola mamishoy en una reunión senti que yo iba encontra del mundo. Que yo era la única que opinaba đistinto. Una embarazada en la mesa. $Y$ otras ya mamás diciendole que al bebé hay que dejarlo llorar que mientras este lleno y limpio no hay más que hacer y del otro lado yo diciendo que es según el bebé. Según como sea el o ella te va dejar o no hacer cosas o va a dormir sólo. Me tuve que callar porque sólo yo opinaba eso

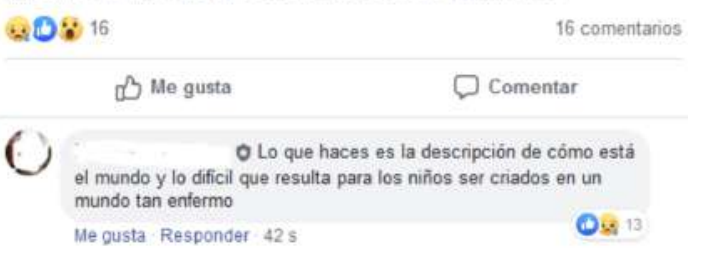

Fuente: publicación en grupo de Facebook, 9/08/2019

Sus posturas alternativas con respecto de visiones más generalizadas o tradicionales les implican una batalla constante con sus entornos familiares directos o políticos. El desahogo con respecto de estos es una muestra. Los comentarios en respuesta son de solidaridad en la actitud y en el plantarse frente a suegras, madres, hermanas, esposos, y todos aquellos que no estén de acuerdo. Dado que su posición se sustenta en discursos de referencia científica, dudosa o no, en el grupo se promueve una insistencia sobre el papel de las participantes en la educación del mundo allende del grupo. La idea es que las perspectivas contrarias se deben a la desinformación, ignorancia o reproducción de patrones dañinos difundidos en generaciones previas por el mundo neoliberal y las compañías capitalistas, por ejemplo, de sucedáneos. Por esta misma razón, unas de las publicaciones más frecuentes son aquellas que comparten de manera orgullosa la duración "prolongada" de su periodo de lactancia. La base científica es la referencia a un cálculo antropológico que afirma que, con respecto a la duración de la lactancia en otros animales, el destete humano debería ocurrir, sin intervención de la cultura, entre los 5 y los 7 años. Recurrentemente aparecen publicaciones con referencia a esto. Cada una de las publicaciones es respondida con una gran cantidad de fotografías de lactancia en niños mayores con la duración del periodo, así como felicitaciones mutuas. Lo mismo pasa cuando se hace referencia a alguna figura pública que ha dado lactancia en el espacio público o algún referente de la medicina que aplaude la lactancia prolongada. Este factor se vuelve tan relevante que muchas de las dudas publicadas inician con la edad del niño, duración de la lactancia y forma del parto antes de exponer el asunto. Y quienes exponen que dieron algún tipo de fórmula a sus hijos incluyen en su discurso algún tipo de justificación o solicitan no ser juzgadas. La lactancia es sólo un ejemplo del significado de la maternidad en este grupo y la exigencia de dedicación principal o absoluta a la crianza por parte de los padres. Como para este caso, las acciones que se promueven también impulsan el mantenimiento extendido del lazo entre madre e hijo. A raíz de esto, en el grupo se menciona con frecuencia la concertación del tiempo de la vida productiva y la doméstica, así como los escases de condiciones para que ocurra. También se discute la división de las labores domésticas, que promueven de forma equitativa. En este aspecto, al contrario de lo que ocurre en el otro grupo, las menciones a los compañeros, aunque exponen mayores exigencias, son referidos sin desdén y burla. No hay memes que ridiculicen su papel y, al contrario, hay reconocimiento a su labor en la crianza. Como se ha expuesto, en este grupo los temas tienden a levantar mayor polémica. La autoridad es el tema del grupo y si la postura responde o no a ella. Cuestiones como el aborto, las vacunas y la escolarización son de los más puntos más álgidos. En general las cuestiones de salud, que se presentan en formato similar al otro grupo, son respondidas con mayor inclinación a tratamientos de medicina alternativa como homeopatía e incluso, curación de empacho. La administración del grupo no interviene en la solución de los conflictos sino para externar su voz experta en contra de la vacunación y a favor de la fisiología. Así, se observa una mediación hacia posiciones menos hegemónicas que, sin embargo, para los miembros del grupo se convierten en uno de los paradigmas que determina la forma en la que enfrentarán uno de los momentos más complicados de la vida humana: la reproducción y la crianza.

\section{Conclusiones}

El ejercicio de análisis que aquí se apunta trata de comprender dos abordajes distantes de una misma preocupación: la crianza y la maternidad. En lo único en lo que coinciden es, precisamente, en ese tema. En todo lo demás, la selección trata de cubrir dos polos de un abanico mucho más amplio que incluye un sinfín de grupos preocupados por compartir experiencias de paternidad. Este estudio colabora a reflexionar el papel de los grupos en 
internet como espacios de discusión, acción e intercambio de significados a partir de nuevas formas de asociación y compromiso en entornos de socialización virtual. Representa una dimensión casi omitida en ambos bandos, para el análisis de comunidades en línea, los de madres que han pasado desapercibidas. Al mismo tiempo, para el análisis de la maternidad, el análisis del comportamiento en línea de las madres y su reflejo en la vida cotidiana ha estado relegado. Especialmente si se considera la escasez de estudios sobre la maternidad que se han realizado desde la comunicación, siendo atendidos principalmente desde las áreas médicas y psicológicas. Entonces, un estudio de este tipo contribuye a la reflexión sobre la socialidad en línea en condiciones de convergencia mediática y globalización. Se construye a partir de pensar en cómo los espacios de socialización digitales posibilitan que las madres perciban que cuentan con un acompañamiento emocional para atravesar la complejidad de la transición a ser madre. Igualmente, da cuenta de los intercambios simbólico que se reconfiguran en un proceso de este calado. Los conceptos de mediación, comunidad interpretativa y cultura resultan pertinentes para comprender un fenómeno de este tipo. De igual forma, la netnografía se muestra como una metodología que atina a dar cuenta de las interacciones sobre de los significados construidos e intercambiados en comunidades de particularidades virtuales. Mediante esta aproximación ha sido posible identificar cómo interactúan los significados compartidos con aquellos más allá de los grupos, con las posturas tradicionales y hegemónicas así como los discursos empleados para negociar con ellos. A partir de este punto se plantea interesante la profundización del análisis al interior de cada uno de los grupos, particularizando y comparando las distintas posturas en todos los temas específicos.

\section{Referencias}

Alonso, M. (2010). Mediación y Construcción de Sentidos: notas en torno a su articulación teórico-metodológica en el estudio de la apropiación de Internet;Mediation and Construction of Meanings: Notes about its Theoretical and Methodological Articulation in the Study of the. Mediaciones sociales, (6), 3-37. https://doi.org/10.520 9/rev_MESO.2010.n6.22290
Asociación Argentina y Latinoamericana de Eutonía (AALE). (s/f). Qué es la Eutonía. Recuperado el 1 de junio de 2020, de http://eutonia.org.ar/

Barrio, N. (2017, marzo 10). Metodología Emmi Pikler: Educación infantil en libertad. Recuperado el 1 de junio de 2020, de Revista Digital INESEM website: https://revistadigital.inesem.es/educacion-socieda d/metodo-pikler/

Bowler, G. M. J. (2010). Netnography: A Method Specifically Designed to Study Cultures and Communities Online. The Qualitative Report, 15(5), 1270-1275.

Capogrossi, M. L., Magallanes, M. L., Socaire, F. (2015). Los desafíos de Facebook. Apuntes para el abordaje de las redes sociales como fuente. Antropología Experimental, (15), 47-63. https://doi.org/10.17561/rae.v0i15.2390

Dalsgaard, S. (2016). The Ethnographic Use of Facebook in Everyday Life. Anthropological Forum, 26(1), 96-114. https://doi.org/10.1080/00664677.2016.1148011

Del Fresno, M. (2011). Netnografía. Recuperado de https: //books.google.com.mx/books?id=SgPur693MjIC\&p $\mathrm{g}=\mathrm{PA} 13 \& \mathrm{dq}=$ observación+participante+netnografía\&s ource $=$ gbs_selected_pages $\&$ cad $=3 \# v=$ onepage $\& q=o b s$ ervaciónparticipantenetnografía\&f=false

Hine, C. (2004). Etnografía virtual. Barcelona: Editorial UOC.

Imbaquingo Pérez, M. I. (2019). Maternidad en red. Comunidades digitales de crianza como espacios de deconstrucción de la identidad en la maternidad. FEMERIS: Revista Multidisciplinar de Estudios de Género, 4(1), 8. https://doi.org/10.20318/femeris.2 019.4564

Jenkins, H. (2009). Confronting the challenges of participatory culture: media education for the 21st century. Cambridge, MA: The MIT Press.

Kozinets, R. V., Hemetsberger, A., Schau, H. J. (2008). The Wisdom of consumer crowds: Collective Innovation in the age of networked marketing. Journal of Macromarketing, 28(4), 339-354. https://doi.org/10 .1177/0276146708325382

Kozinets, R. V. (1998). On Netnography: Initial Reflections on Consumer Research Investigations of Cyberculture. NA - Advances in Consumer Research, 25, 366-371.

Kozinets, R. V. (2010). Netnography: ethnographic research in the age of the internet (1st ed). Thousand Oaks, CA: Sage Publications Ltd.

Martín-Barbero, J. (1987). De los medios a las mediaciones: comunicación, cultura y hegemonía. México: Ediciones G. Gili.

McDaniel, B. T., Coyne, S. M., Holmes, E. K. (2012). New mothers and media use: Associations between blogging, social networking, and maternal well-being. Maternal and Child Health Journal, 16(7), 1509-1517. https: //doi.org/10.1007/s10995-011-0918-2 
Moreno, M. (2020, abril 30). Facebook supera los 2.600 millones de usuarios activos. Recuperado el 30 de mayo de 2020, de TreceBits. Redes sociales y tecnología website: https://www.trecebits.com/2020/04/30/fac ebook-supera-los-2-600-millones-de-usuarios/

Navarro Casillas, A. M. (2016). Comunidades ecosociales de mujeres en la red. Detonante para repensar la ciudadanía en el contexto global. Caleidoscopio - Revista Semestral de Ciencias Sociales y Humanidades, 69. https://doi.or $\mathrm{g} / 10.33064 / 0 \mathrm{crscsh} 121$

Nor, N. M., Othman, N., Yusof, S. A. M. (2018). A conceptual understanding of health virtual Communities. International Conference on ICT for Transformation, 0-5. Recuperado de http://unisep.lib.unishams.edu.my /xmlui/bitstream/handle/123456789/12607/ArticleICT 4T.pdf?sequence $=1 \&$ is Allowed $=y$

Orozco, G. (1991). La audiencia frente a la pantalla. Una exploración del proceso de recepción televisiva. Diálogos de la Comunicación, 30, 54-63. Recuperado de $\mathrm{http} / / / \mathrm{ccdoc}$.iteso.mx/cat.aspx? $\mathrm{cmn}=$ browse $\& \mathrm{id}=1607$

Orozco, G. (2000). Travesías y desafíos de la investigación en América Latina. Comunicación y Sociedad, (38), 11-36.

Orton-Johnson, K. (2017). Mummy blogs and representations of motherhood: "Bad mummies" and their readers. Social Media and Society, 3(2). https://doi.org/10.1 177/2056305117707186

Redacción SinEmbargo. (2020, febrero 3). Facebook, la red social preferida en México que puede provocar ansiedad y desinformación. SinEmbargo.mx. Recuperado de https: //www.sinembargo.mx/03-02-2020/3724136

Rheingold, H. (2000). The Virtual Community: Homesteading on the Electronic Frontier (2nd ed.). Reading, Massachusetts: Addison-Wesley.

Ruiz, M. À. (2008). Ciberetnografía: comunicad y territorio en el entorno virtual. En E. Ardèvol, A. Estalella, D. Domínguez (Eds.), La mediación tecnológica en la práctica etnográfica (pp. 117-132). Recuperado de http://www.ankulegi.org/5-la-mediacion-tecnolog ica-en-la-practica-etnografica/

Statista. (2020, febrero 3). Mexico: number of Facebook users 2019 I Statista. Recuperado el 31 de mayo de 2020, de https://www.statista.com/statistics/282326/number- of -facebook-users-in-mexico/

Turpo, O. W. (2008). La netnografía: un método de investigación en Internet. Educar, 42, 81-92.

Villanueva Aburto, D. (2017). La maternidad glorificada: análisis de los discursos sobre maternidad de mujeres que participan en grupos de crianza respetuosa de la región de Valparaíso, Chile. Revista Punto Género, (7), 138-155. https://doi.org/10.5354/0719-0417.2017.462 72 